\title{
Tratamiento adyuvante en pacientes diagnosticados de carcinoma no microcítico de pulmón: estado de la evidencia científica
}

\author{
E. Esteban, N. Villanueva, I. Muñiz, M. Luque, P. Jiménez, B. Llorente, M. Capelan, P. Blay, J. Fra, \\ Y. Fernández, J. M. Vieitez, E. Estrada, J. M. Buesa, Á. Jiménez Lacave
}

\section{Resumen}

La cirugía representa el tratamiento curativo de elección para los pacientes que son diagnosticados de carcinoma no microcítico de pulmón en estadio local. Sin embargo, gran parte de ellos experimentan una recurrencia de su enfermedad lo que ha llevado al empleo de opciones terapéuticas tanto locales como sistémicas con el ánimo de mejorar las posibilidades de curación. Durante las últimas décadas diversos estudios comparativos heterogéneos y varios meta-análisis publicados en los años 95 y 97 no demostraron de forma significativa una ventaja al asociar la quimioterapia y/o radioterapia adyuvante a la cirugía. Desde entonces y hasta nuestros días otros trabajos realizados con un número mayor de pacientes y empleando una quimioterapia más eficaz basada en combinaciones de platino, coinciden en describir un beneficio con su utilización aunque no de forma unánime. Más recientemente, un nuevo meta-análisis recopilando la mayoría de los estudios antes mencionados ha confirmado de forma significativa una mejoría absoluta del $4 \%$ en la supervivencia global de los pacientes sometidos a quimioterapia adyuvante, especialmente en los estadios patológicos II-III tras cirugía y tratados con regímenes que incluyen cisplatino y vinorelbina. Queda por ser determinado el papel que desempeñan otros agentes como el uracilo/tegafur así como la radioterapia en el contexto adyuvante.

Palabras clave: Carcinoma no microcítico de pulmón. Adyuvancia.

Oncología, 2006; 29 (9):368-377 


\section{Summary}

Surgery is the current treatment of choice in patients with early-stage non-small cell lung cancer. Based on the high rates of recurrence, additional local and systemic treatments have been developed, aimed at improving the cure rates. The comparative studies about the benefits of post-operative adjuvant chemotherapy and/or radiotherapy, and the meta-analysis studies made during the last decades, reviewed in some articles appeared in 95 and 97, did not confirm a significant improvement of the overall survival. Since then, new comparative trials carried out with a higher number of patients and with more active and standard chemotherapy seem to show a benefit of the administration of platinum-based chemotherapy, although it has not gained general acceptance. More recently, a new meta-analysis, that included the previous studies, has confirmed an overall increase of $4 \%$ in survival of patients treated by surgery and adjuvant chemotherapy, especially in stages II-III patients receiving schedules of cisplatin and vinorelbine. Further studies are needed to determine the real therapeutic value of other agents, as uracil-tegafur and radiotherapy.

Key words: Lung cancer. Surgery. Adjuvant treatment. Chemotherapy. Radiotherapy.

\section{Introducción}

El carcinoma no microcítico de pulmón (CNMP) representa más del $75 \%$ de todas las neoplasias que se originan en el pulmón siendo además responsable del $30 \%$ de todas las muertes por cáncer en nuestra sociedad. Cuando es posible por su localización, la cirugía constituye la opción terapéutica de elección con intención curativa, aunque solo un tercio de los casos en el momento de su diagnóstico van a tener un estadio localizado que permita dicho tratamiento. A pesar del esfuerzo quirúrgico, muchos pacientes operados por un CNMP van a desarrollar una recurrencia local o a distancia de la enfermedad debido a la existencia de micro-metástasis no detectables en el momento de la cirugía ${ }^{1}$. Esos resultados han sido el motivo por el que durante las últimas décadas se llevaran a cabo múltiples estudios en la investigación clínica empleando diferentes modalidades terapéuticas sistémicas incluyendo la inmunoterapia y quimioterapia, así como la radioterapia adyuvante a la cirugía con la intención de mejorar las posibilidades de controlar la enfermedad y que a continuación pasamos a describir.

\section{Quimioterapia y radioterapia}

\section{Estudios iniciales}

Basados en el concepto anteriormente expuesto de la existencia de micro-metástasis no detectable en el momento de la cirugía, la utilización de la quimioterapia adyuvante ha representado una forma lógica para intentar su erradicación. Ya desde la década de los años sesenta, diversos tipos de tratamiento sistémico utilizando inmunoterapia inespecífica y/o agentes citotóxicos tales como alquilantes, antraciclinas y cisplatino fueron desarrollados con resultados contradictorios en su repercusión sobre la supervivencia libre de recurrencia y global. En el año 1995 se publicó un meta-análisis llevado a cabo por el NSCLC Collaborative Group en el que se analizaron 52 estudios realizados desde 1965 hasta 1991 comparando la cirugía sola o acompañada de quimioterapia adyuvante (4.357 pacientes en total). Los datos demostraron que los agentes alquilantes se relacionaban con una reducción en un $15 \%$ de la supervivencia (HR 1,2). Por el contrario, al analizar 8 estudios incluyendo 1.394 pacientes tratados con 


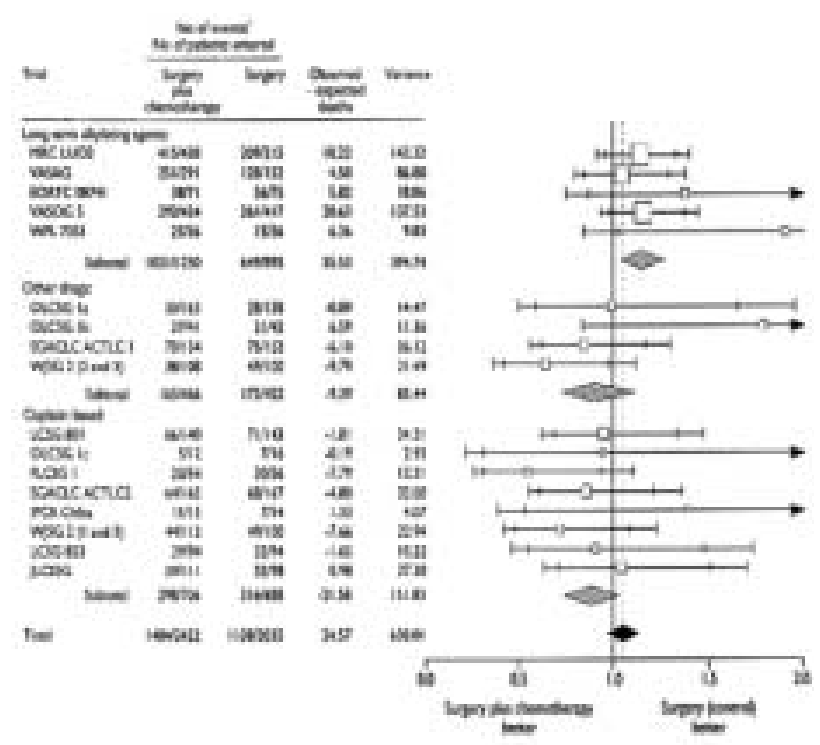

Figura 1. Meta-análisis NSCLC Collaborative Group 1995.

esquemas de cisplatino se describió un beneficio absoluto del $5 \%$ en la supervivencia a los 5 años favorable para el grupo tratado con cisplatino adyuvante. Dicho efecto beneficioso no alcanzó una significación estadística (HR 0,87; p=0,08) por lo que en ese momento no se consideró como estándar la quimioterapia (Figura 1). De forma adicional, en el mismo meta-análisis la asociación de la radioterapia y quimioterapia comparada con la administración solo de la radioterapia como adyuvancia, ofrecía una reducción en un $6 \%$ en el de riesgo de muerte sin llegar a ser una ventaja significativa $(\mathrm{p}=0,48)^{2}$.

Independientemente de esos datos, los pacientes con márgenes quirúrgicos positivos, resección incompleta del tumor o afectación ganglionar son considerados de riesgo elevado para la recidiva y en ellos, la utilización de la radioterapia ha sido considerada como una herramienta eficaz para mejorar el control local de la enfermedad. Un meta-análisis publicado en el año 1997 (PORT meta-analysis trialist group) incluyendo 9 estudios (2.128 pacientes) que comparaban radioterapia post-cirugía vs observación en estadios patológicos I-II y III, describió una reducción del $21 \%$ en la supervivencia (HR 1,2) con la utilización de la radioterapia, con un claro perjuicio para el estadio I (N0), menos en estadio II y sin efecto beneficioso o perjudicial para el estadio III (HR 1) ${ }^{3}$. A pesar de que se relacionaran con la utilización de técnicas y dosis antiguas e inadecua- das, dichos resultados han sido la base para que hasta el momento el papel de la radioterapia adyuvante en el CNMP tras cirugía completa con márgenes adecuados, sea considerado un tema en discusión.

Tras todos esos resultados, el avance científico en cuanto al papel de la quimioterapia adyuvante se puede dividir en dos vías de investigación clínica; Una la desarrollada en pacientes asiáticos (Japón) utilizando fluoropirimidinas y otra la llevada a cabo en el mundo occidental basada en la utilización de combinaciones de platino con agentes de segunda y tercera generación.

\section{Estudios actuales de quimioterapia adyuvante con uracilo/tegafur (UFT)}

El fármaco UFT es una fluoropirimidina desarrollada en Japón que cuenta entre sus características con una tolerancia oral buena así como una hipotética propiedad anti-angiogénica. Esto ha facilitado que al menos se hayan llevado a cabo 6 estudios comparativos en pacientes asiáticos (la mayoría de los casos con estadios patológicos I) a los que se les asignó de forma aleatoria a recibir o no $400 \mathrm{mg}$ orales de UFT durante 1 o 2 años tras la cirugía. En tres de ellos se describió una ventaja significativa en la supervivencia global a favor del grupo tratado con $\mathrm{UFT}^{4-6}$ aunque en los otros tres estudios no se confirmó dicho beneficio ${ }^{7-9}$. Un meta-análisis posterior recopilando los datos de 2.000 pacientes incluidos en todos esos trabajos y con una mediana de seguimiento de 5 años parece confirmar un aumento absoluto de un $5 \%$ en la supervivencia global a los 7 años (82\% versus $77 \%$ ) a favor del tratamiento con UFT (HR 0,74 [0,61-0,88] p = 0,001) $)^{10}$ (Figura 2). De esos resultados se puede desprender que los

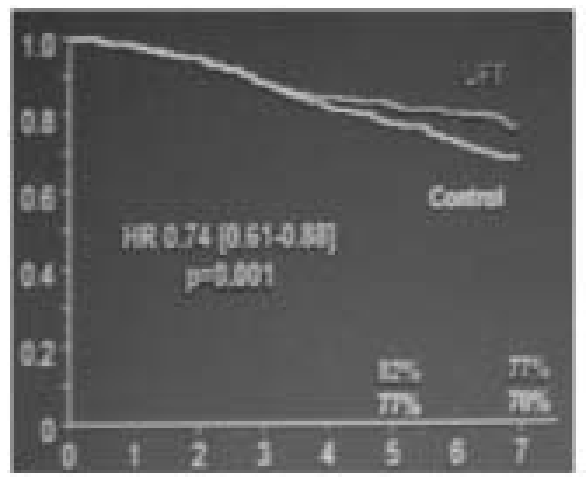

Figura 2. Meta-análisis de UFT ${ }^{10}$. 
TABLA I

Estudios comparativos actuales de quimioterapia adyuvante y supervivencia

\begin{tabular}{|c|c|c|c|c|c|c|}
\hline Estudio & Estadio & Esquema & Control & $N^{o}$ Pacientes & $H R(p)$ & $95 \% I C$ \\
\hline Meta-análisis 1995 & & Cis/asociación & Observación & 1394 & $0,87(0,08)$ & $0,7-1$ \\
\hline INT 0116 & T1-3 N1-2 & Cis/VP x $4+\mathrm{RT}$ & Radioterapia & 462 & $0,93(0,56)$ & \\
\hline BLT & I-II-IIIA & $\begin{array}{l}\text { Cis/Vinca o } \\
\text { MIC/MVP x } 3\end{array}$ & Observación & 381 & $1(0,9)$ & \\
\hline ALPI & I-II-IIIA & MVC x 3 & Observación & 1088 & $0,96(0,58)$ & $0,8-1,1$ \\
\hline IALT & I-II-IIIA & $\begin{array}{c}\text { Cis/VP o } \\
\text { Vinca x 3-4 }\end{array}$ & Observación & 1867 & $0,86(0,03)$ & $0,76-0,98$ \\
\hline NCI-BR10 & $\begin{array}{l}\text { T2-N0 } \\
\text { T1-2 N1 }\end{array}$ & $\begin{array}{c}\text { Cis/Vinorelbina } \\
\text { x } 4\end{array}$ & Observación & 640 & $0,7(0,03)$ & $0,52-0,91$ \\
\hline CALGB 9633 & IB & $\begin{array}{c}\text { Carbo/Paclitaxel } \\
\text { x } 4\end{array}$ & Observación & 344 & $0,8(0,1)$ & $0,6-1,07$ \\
\hline ANITA I & I-II-IIIA & Cis/Vinorelbina $\mathrm{x} 4$ & Oservación & 840 & $0,7(0,015)$ & $0,66-0,95$ \\
\hline $\begin{array}{l}\text { Meta-análisis } \\
\text { LACE }\end{array}$ & I-II-IIIA & $\begin{array}{l}\text { Combinacines de } \\
\text { Cisplatino x 3-4 }\end{array}$ & Observación & 4584 & $0,89(<0,005)$ & $0,82-0,96$ \\
\hline
\end{tabular}

Cis: cisplatino; Vinca: alcaloides de la vinca; MIC: mitomicina+ifosfamida+cisplatino; RT: radioterapia; Carbo: carboplatino.

MVC: mitomicina+vindesina+cisplatino

pacientes asiáticos, especialmente mujeres con adenocarcinoma y con estadio I con un tamaño del tumor superior a los dos centímetros se benefician de la administración de UFT de forma adyuvante. No obstante, los puntos en debate sobre la utilización de este medicamento se relacionan entre otros con aclarar el motivo por el que las curvas de supervivencia no se separan antes de los 4 años de seguimiento o incluso conocer el efecto real del fármaco en la población no asiática.

\section{Estudios actuales de quimioterapia adyuvante utilizando esquemas de platino}

Tras la publicación del primer meta-análisis del NSCLC Collaborative Group en el año 1.995, se han llevado a cabo al menos 7 estudios comparativos utilizando de regímenes de platino (Tabla I).

El primero de ellos publicado por el North American Intergroup Trial (INT 0116) analizó en 462 pacientes con estadio patológico T1-3N1-2 la administración adyuvante de radioterapia sola o asociada de forma concurrente con cisplatino y VP-16. Los hallazgos no demostraron diferencias entre los dos grupos de tratamiento en supervivencia (HR 0,93; $\mathrm{p}=0.56$ ) ni en los patrones de recurrencia, aunque el diseño y poder estadístico no parecen ser adecuados ni tampoco el brazo control utilizado con radioterapia $^{11}$.

Por otra parte, un trabajo realizado en Gran Bretaña y denominado Big Lung Trial (BLT) investigó el papel de la quimioterapia adyuvante o neo-adyuvante en pacientes con estadio I a IIIA tratados bien con cirugía, radioterapia o el mejor cuidado de soporte $(n=1.394)$. Las combinaciones de quimioterapia utilizadas incluían cisplatino asociado con mitomicina y/o alcaloides de la vinca de segunda generación. En el análisis del grupo de pacientes tratados con quimioterapia de forma adyuvante no se observo diferencias en la supervivencia con respecto a cirugía sola (HR 1; $=0,9)$ aunque una vez más el diseño inadecuado y el poder estadístico bajo ( $\mathrm{n}=381 \mathrm{pa}-$ cientes) no permiten sacar conclusiones definitivas ${ }^{12}$.

Otro estudio más amplio y con un diseño mejor, denominado Adjuvant Lung Project Italy (ALPI) incluyó a pacientes con estadio patológico I a IIIA asignándoles de forma aleatoria a recibir o no 3 ciclos de quimioterapia con la combinación de mitomicina, vindesina y cisplatino (MVP) quedando a elección del investigador la administración de ra- 
dioterapia adyuvante. El objetivo principal era confirmar una mejoría en la supervivencia a los 5 años del 7\% (57\% vs 50\%) para lo cual se necesitaba incluir un total de 1.200 pacientes. Con una mediana de seguimiento de 65 meses y con 1.088 pacientes analizados, se describió un $1 \%$ de mejoría en la supervivencia a favor de la quimioterapia (HR 0.96; $\mathrm{p}=0,58)^{13}$. Diversos motivos han sido sugeridos como responsables de la ausencia de beneficio entre ellos, la inclusión de pacientes con estadio IA, la utilización de la mitomicina-C y radioterapia (ambos con un hipotético efecto adverso sobre la supervivencia) o que dos tercios de los pacientes no pudiesen recibir el total de la quimioterapia planificada debido a la toxicidad registrada.

El Internacional Adjuvant Lung Cancer Trial (IALT) ha sido otro de los estudios publicados con mayor número de pacientes y con más largo seguimiento. Dicho trabajo tenía como objetivo principal confirmar un incremento en un 5\% de la supervivencia global con la incorporación de la quimioterapia adyuvante (55\% versus 50\%). Los esquemas empleados incluían combinaciones de cisplatino asociado a VP-16 o alcaloides de la vinca administrados en un número de 3 o 4 ciclos tras la cirugía. El cálculo inicial para cumplir la hipótesis de trabajo se hizo en base a la inclusión de 3.300 pacientes con estadio patológico I, II y IIIA, aunque por razones de dificultades en el reclutamiento, el ensayo se detuvo al alcanzar un número de 1.800 casos. Con una mediana de seguimiento de 56 meses, este ha sido el primer trabajo que describió una ventaja significativa, aunque del $4 \%$ en la supervivencia global a los 5 años con la incorporación de la quimioterapia adyuvante (44\% versus $40 \%$; HR 0,86 ; $\mathrm{p}=0,03)^{14}$. A diferencia del ALPI, solamente el $25 \%$ de los pacientes recibieron radioterapia adyuvante y ninguno de los esquemas permitidos incluyeron la mitomicina como posibles causas de la ventaja observada. No obstante, hay que subrayar que el IALT no cumplió en realidad su objetivo principal ni en el incremento de la supervivencia ni en el número de pacientes necesarios para su análisis, por lo que el poder estadístico se podría considerar por debajo del $80 \%$ planeado previamente. De forma adicional, en un análisis por subgrupos solo aquellos pacientes con estadio IIIA y los que fueron tratados con el esquema de cisplatino y vinorelbina parecen ser los que se benefician de forma significativa.
Desde la publicación del IALT, otros tres estudios comparativos empleando combinaciones de quimioterapia de tercera generación administrados de forma adyuvante a la cirugía han sido dados a conocer con resultados favorable en casi todos ellos. El primero, llevado a cabo por el National Cancer Institute of Canada (NCIC-JBR.10) utilizó el esquema de cisplatino y vinorelbina (4 ciclos) en pacientes con estadio patológico IB a IIB tras cirugía. El objetivo principal era demostrar una mejoría en la supervivencia a los 3 años del $10 \%$ al incorporar la quimioterapia a la cirugía (60\% versus $50 \%$ ), con un poder estadístico del $80 \%$ y un nivel de significación unilateral del $5 \%$ para lo cual se requería la inclusión de 640 pacientes. Con un registro de 532 pacientes y un análisis de 482 casos, el estudio ha sido publicado describiendo un beneficio del $15 \%$ a los 5 años en las curvas actuariales de supervivencia (69\% versus 54\%; HR 0,7; $\mathrm{p}=0,03$ ). Por subgrupos, solo los pacientes con estadio II parecen beneficiarse de forma significativa ${ }^{15}$. Un dato adicional en ese tipo de sub-análisis, mantiene el efecto beneficioso de la adyuvancia en los pacientes con edad superior a los 65 años (hasta los 74 años) a pesar de recibir una dosis inferior de quimioterapia a la programada de inicio $^{16}$.

El segundo trabajo comparativo, en este caso firmado por el Cancer and Leukemia Group B (CALG B- 9633) fue planteado en pacientes con estadio IB quirúrgico asignados de forma aleatoria a recibir o no 4 ciclos de la combinación de carboplatino/Paclitaxel tras cirugía ${ }^{17}$. Con una mediana de seguimiento de 34 meses y con la inclusión de 344 pacientes de los 504 planificados inicialmente debido a un lento reclutamiento, los autores presentaron unos resultados iniciales favorables a la quimioterapia al incrementar un $12 \%$ las curvas de supervivencia a los 4 años (71\% versus 59\%; HR 0,6; p=0,03). Sin embargo, en una actualización posterior y con una mediana de seguimiento de 54 meses, se ha perdido la diferencia significativa en la supervivencia a los 5 años entre los dos grupos de pacientes (60\% versus $57 \%$; HR 0,$8 ; \mathrm{p}=0,10)^{18}$. Tanto el JBR.10 como el CALG B-9633 coinciden en diversas variables como posible causa de los resultados favorables a la administración de quimioterapia tales como no haber permitido la administración de la radioterapia adyuvante, la presencia de adenocarcinoma como histología más frecuente (60\% de los casos), así co- 
mo que un tercio de los pacientes incluidos correspondían al sexo femenino.

El último de los estudios a favor de la quimioterapia ha sido el denominado Adjuvant Navelbine International Trialist Association trial (ANITA) en el que 840 pacientes con estadios IB a IIIA tras cirugía fueron asignados a recibir o no 4 ciclos de quimioterapia adyuvante con la combinación de cisplatino/vinorelbina. Con esa muestra se pretendía como objetivo principal, confirmar un incremento del $10 \%$ en la supervivencia a los 2 años con un poder estadístico del $90 \%$. Los resultados fueron presentados en el congreso mundial de pulmón y en el de la sociedad americana de oncología médica del año 2.005 describiendo con una mediana de seguimiento de 70 meses una ventaja significativa a favor de la quimioterapia en supervivencia del $51,2 \%$ versus $42,6 \%$ (HR 0,7; $\mathrm{p}=0,015)$. En el análisis por subgrupos, solo los pacientes con estadios II y III mostraron una mejoría significativa. De forma adicional, en los casos con afectación ganglionar mediastínica (N2) la asociación de radioterapia y quimioterapia parece tener un impacto favorable en la supervivencia con respecto a aquellos tratados solo con quimioterapia (47\% versus $34 \%$ ), distinto a lo observado cuando solo hay afectación ganglionar en el hilio pulmonar (N1) en los que la incorporación de radioterapia podría ocasionar un efecto adverso (34\% versus $40 \%)^{19}$.

Un meta-análisis reciente, llamado Lung Adjuvant Cisplatin Evaluation (LACE) ha recopilado los datos de 4.584 pacientes incluidos en 5 de los estudios mencionados previamente (ALPI, IALT, BLT, JBR10 y ANITA) que utilizaron esquemas de cisplatino (no incluyeron el estudio CALG B 9633 por emplear carboplatino) presentando sus hallazgos en el último congreso de la sociedad americana de oncología médica (ASCO 2006). Con una mediana de seguimiento de 5 años, se confirma un beneficio absoluto del $4,2 \%$ en la supervivencia a favor de la quimioterapia adyuvante (HR 0,89; 95\% IC: 0,820,96; $\mathrm{p}<0,005$ ) (Figura 3), siendo significativamente favorable para los pacientes con estadios II y III (HR 0,83; 95\% IC: 0,73-0,95) pero no para los estadios IA (HR 1,4; 95\% IC: 0,96-2,09) o IB (HR 0,92; 95\% IC: 0,78-1,1) (Figura 4). Del mismo modo, solamente los esquemas de cisplatino con vinorelbina demuestran una asociación beneficiosa con la supervivencia respecto a otras combinaciones (HR 0,80; 95\% IC: 0,7-0,9; P=0,04) (Figura 5). En

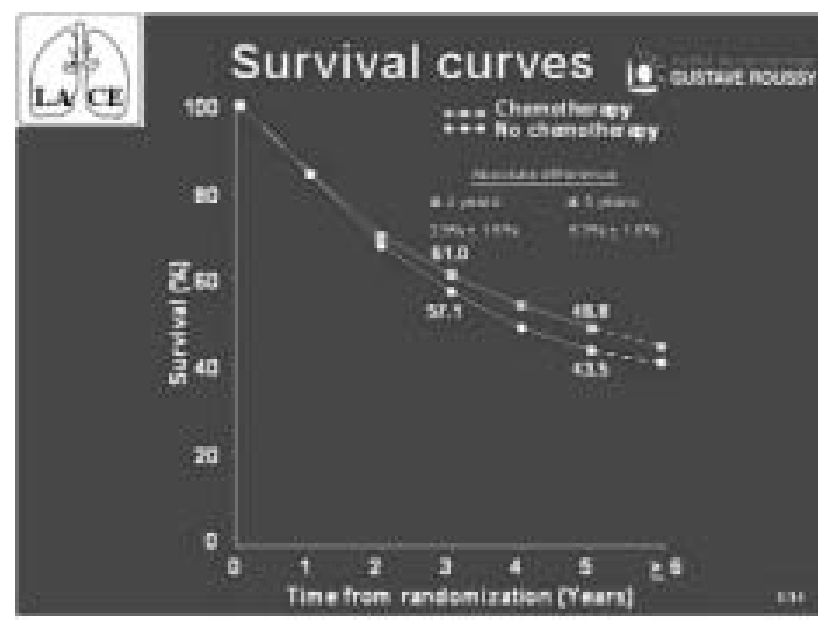

Figura 3. Meta-análisis LACE. Curvas de supervivencia.

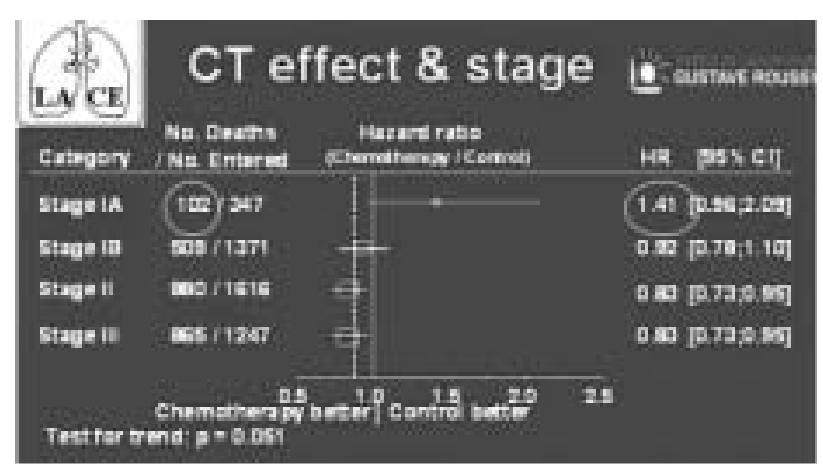

Figura 4. Meta-análisis LACE. Resultados según estadios de la enfermedad.

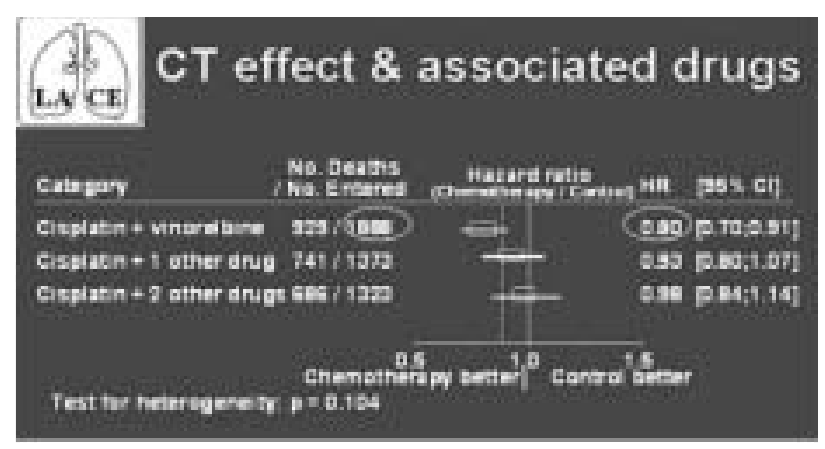

Figura 5. Meta-análisis LACE. Resultados por tipo de quimioterapia.

los datos expuestos también se hace referencia a la ausencia de interacción de la quimioterapia con el sexo, edad, radioterapia o dosis total de cisplatino administrada ${ }^{20}$.

De forma general, los resultados en la mayoría de los estudios revisados incluyendo el meta-análisis 
TABLA II

Estudios comparativos con quimioterapia adyuvante. Características de los pacientes

\begin{tabular}{|c|c|c|c|c|c|}
\hline Características & $A L P I$ & $I A L T$ & NCI-C BRIO & CALGB 9633 & ANITA I \\
\hline $\mathrm{N}^{\circ}$ Pacientes QT/Control & $472 / 465$ & $932 / 935$ & $243 / 239$ & $173 / 171$ & $433 / 407$ \\
\hline Estratificación & - & $\begin{array}{c}\text { Centro } \\
\text { Tipo Cirugía } \\
\text { Estadio Patolológico }\end{array}$ & $\begin{array}{c}\text { N0 vs N1 } \\
\text { K-Ras (+) vs (-) }\end{array}$ & $\begin{array}{l}\text { Escamoso vs No } \\
\text { Mediastinoscopia } \\
\text { Si vs No }\end{array}$ & $\begin{array}{c}\text { Centro } \\
\text { Estadio } \\
\text { Histología }\end{array}$ \\
\hline Mediana edad & 61 & 59 & 61 & 61 & 59 \\
\hline Sexo femenino (\%) & 14 & 19 & 34 & 36 & 14 \\
\hline \multicolumn{6}{|l|}{$\begin{array}{l}\text { Tipo Disección } \\
\text { Ganglionar (\%) }\end{array}$} \\
\hline Simple/Completa & $43 / 57$ & No referida & No referida & No referida & No referida \\
\hline \multicolumn{6}{|l|}{ Estadio patógico (\%) } \\
\hline I & 39 & 36 & 46 & 100 (IB) & 36 \\
\hline II & 31 & 25 & 54 & & 29 \\
\hline III & 29 & 39 & & & 35 \\
\hline \multicolumn{6}{|l|}{ Histología (\%) } \\
\hline Escamoso & 51 & 46 & 38 & 39 & 60 \\
\hline No Escamoso & 49 & 54 & 62 & 61 & 40 \\
\hline Neumectomia (\%) & 24 & 35 & 25 & 11 & 38 \\
\hline \multicolumn{6}{|l|}{ Estado General } \\
\hline 0 & - & 54 & 49 & 51 & 95 \\
\hline I & - & 38 & 51 & 49 & 90 \\
\hline
\end{tabular}

QT: quimioterapia

LACE, coinciden en confirmar que la quimioterapia adyuvante beneficia a un restringido número de pacientes tras ser sometidos a la cirugía, independientemente de la heterogeneidad en las características clínicas y métodos quirúrgicos empleados (por ejemplo, solo en el ALPI se hace referencia al tipo de disección linfática mediastínica realizada, dato que puede tener valor terapéutico y pronóstico) (Tabla II). Además, la quimioterapia parece ocasionar una toxicidad que debe ser tenida en consideración, especialmente al ser aplicada a pacientes sometidos a una cirugía importante (aquellos con neumectomia) y que delimita su indicación solo para los casos que cuentan con un buen estado funcional. Dicha toxicidad ha sido descrita de forma predominante como hematológica (incluyendo un 1\% de muertes tóxicas) y que obliga a una reducción de las dosis y/o número de ciclos a ser administrados en cerca del $50 \%$ de los casos, dato en el que coinciden la mayoría de los estudios revisados (Tabla III).

Por todos esos motivos tanto de toxicidad como por el restringido beneficio, se están llevando a ca- bo de forma retrospectiva y prospectiva análisis sobre características biológicas más específicas que permitan delimitar mejor los pacientes que se benefician de un tratamiento adyuvante. En ese sentido, el ALPI no encontró ninguna relación con sus resultados y la determinación de Ki-67, P53 o $\mathrm{K}-\operatorname{Ras}^{13}$. La presencia de mutación de esta última proteína (K-Ras) se ha demostrado que está presente en el $23 \%$ de las muestras analizadas en el estudio NCI-BR10, aunque no es la forma mutada sino la salvaje la que parece guardar una relación predictiva de beneficio a la quimioterapia adyuvante $^{15}$. De forma más reciente, el IALT en un análisis inmunohistoquímico de la proteína ERCC1 (excision repair cross-complementing 1) ha confirmado su presencia en el 44\% de 783 muestras analizadas, sugiriendo que tiene un valor predictivo independiente. En ese sentido, solo aquellos pacientes con ausencia de ERCC1 en su tumor parecen beneficiarse de la administración de quimioterapia adyuvante con cisplatino cuando se comparan con los que no la recibieron. Sin embargo ese 
TABLA III

Estudios comparativos con quimioterapia adyuvante. Esquema, dosis y toxicidad

\begin{tabular}{|c|c|c|c|c|c|}
\hline Características & $A L P I$ & $I A L T$ & NCI-C BR10 & CALGB 9633 & ANITA I \\
\hline Esquema QT (\%) & MVC & $\begin{array}{c}\text { Cis/VP (56) } \\
\text { Cis/VNB (27) } \\
\text { Cis/Vbl (11) } \\
\text { Cis/Vds (6) }\end{array}$ & $\mathrm{Cis} / \mathrm{VNB}$ & Carbo/paditaxel & Cis/VNB \\
\hline $\mathrm{N}^{\mathrm{o}}$ de ciclos & 3 & $3-4$ & 4 & 4 & 4 \\
\hline Dosis Cisplatino $\left(\mathrm{mg} / \mathrm{m}^{2}\right)$ & 100 & $80-120$ & 100 & AUC 6 & 100 \\
\hline Intensidad de dosis & 33,3 & $25-30$ & 25 & & 30 \\
\hline Tratamiento Completo (\%) & 56 & $76 \geq 2$ ciclos & 49 & 55 & \\
\hline Radioterapia Post (\%) & 43 vs 43 & 23 vs 28 & No & No & Sí \\
\hline Mediana de dosis & 50-54 Gy & $50 \mathrm{~Gy}$ & & & Sin especificar \\
\hline Toxicidades (\%) & & & & & \\
\hline Neutropenia 3/4 & 28 & 17 (G4) & 73 & 36 & 86 \\
\hline Infección/Neutrop Febril & & - & 7 & 5 & 8.5 \\
\hline Muerte Tóxica & 3 pts $(0,5)$ & 7 pts $(0,8)$ & 2 pts $(0,9)$ & 0 & 5 pts (1) \\
\hline
\end{tabular}

Cis: cisplatino; VNB: vinorelbina; Vbl: vinblastina; Vds: vindesina; pts: pacientes; Carbo: carboplatino; MVC: mitomicina + vindesina + cisplatino.

beneficio o diferencia no se confirmó entre los que cuentan con la presencia de ERCC $1^{21}$. En ese mismo estudio y también de una forma retrospectiva no se vio relación predictiva o pronostica con otras determinaciones tales como P53, BCL2, telomerasa, EGFR o AKT.

\section{Nuevas estrategias de tratamiento adyuvante}

En la actualidad existen datos obtenidos en el tratamiento de pacientes con CNMP en estadio avanzado con la incorporación de agentes diseñados para ejercer su efecto antitumoral de forma específica sobre estructuras proteicas claves para la proliferación de la célula tumoral. De entre ellos destacan las pequeñas moléculas y anticuerpos monoclonales desarrollados para bloquear receptores de factores de crecimiento tanto epidérmico (EGFR) como endotelial (VEGFR). En base a esa experiencia, un estudio del intergrupo americano plantea incluir a 1.500 pacientes con estadio patológico IA a IIIB para ser asignados de forma aleatoria a recibir 4 ciclos de quimioterapia de tercera generación (aceptan la combinación de carboplatino/paclitaxel) asociado o no con el anticuerpo monoclonal anti-VEGFR "bevacizumab" a una dosis de $15 \mathrm{mg} / \mathrm{m}^{2}$ cada 3 semanas durante un año. El objetivo principal del estudio será detectar una diferencia del $25 \%$ en la mediana de supervivencia y con un poder estadístico del $85 \%$ (66 meses versus 83,5 meses). En cuanto a la utilización de pequeñas moléculas inhibidoras de EGFR como tratamiento adyuvante, existe un trabajo publicado por el SWOG (S-0023) comparando gefitinib administrado a pacientes con estadio III después de haber sido tratados con quimioterapia y radioterapia radical $^{22}$. Los datos preliminares han expuesto una peor supervivencia en el grupo que recibió gefitinib con respecto a placebo, obligando con ello a cerrar otro estudio ideado por el NCI de Canadá empleando el mismo fármaco como adyuvancia.

Otra posible alternativa como tratamiento adyuvante de los pacientes con CNMP tras cirugía se base en un concepto inmunológico ya explorado durante décadas al utilizar agentes estimuladores inespecíficos con la intención de destruir o controlar la enfermedad residual microscópica mínima, sin que se haya demostrado un beneficio. No obstante, en la actualidad dicha modalidad terapéutica ha cobrado interés al disponer de diversas vacunas desarrolladas contra antígenos tumorales específicos. Entre ellas, destacan las vacunas BLP25 ${ }^{23}$ y MAGE-A3 ${ }^{24}$ que están siendo investigadas ya en el marco de un tratamiento adyuvante. 


\section{Tratamiento neo-adyuvante}

La administración de un tratamiento previo a la cirugía o neo-adyuvante alberga una serie de hipotéticas ventajas tales como poder ser aplicado a pacientes que cuentan con mejor estado general y por tanto con mayores posibilidades de tolerar y recibir la totalidad del tratamiento así como poder valorar de forma objetiva la sensibilidad del tumor al tratamiento aplicado e incluso conseguir disminuir el tamaño tumoral facilitando la actuación local quirúrgica. Entre las modalidades terapéuticas, la quimioterapia neo-adyuvante es la que ha tenido un mayor desarrollo clínico aunque con resultados contradictorios que han impedido que alcanzara un protagonismo como el adquirido por la quimioterapia adyuvante. En ese sentido, un estudio comparativo francés (French Thoracic Cooperative Group) no demostró ventajas en la supervivencia global con la administración de 2 ciclos de cisplatino, mitomicina e ifosfamida con respecto a cirugía sola en 355 pacientes con estadio temprano de la enfermedad (I a IIIA). El ese trabajo se describió un $65 \%$ respuestas objetivas y una mejoría en variables como supervivencia libre de recurrencia y metástasis a distancia con la quimioterapia neo-adyuvante. En análisis por subgrupos también se observó una mejoría en la supervivencia de los casos con estadio IB-II tratados con la quimioterapia ${ }^{25}$. Por esos motivos, el SouthWest Oncology Group (estudio 9900) comenzó un estudio comparativo utilizando la combinación de carboplatino/paclitaxel (3 ciclos) como tratamiento neo-adyuvante a la cirugía en pacientes con estadio $\mathrm{T} 2 \mathrm{~N} 0$ a T3N1 ${ }^{26}$. Ciento setenta y cuatro pacientes fueron asignados a cirugía de entrada y 180 a recibir dicho tratamiento previamente a ser operados y a pesar de sugerir alguna ventaja en este último grupo, el trabajo ha sido cerrado al adoptarse la quimioterapia adyuvante como tratamiento estándar en este tipo de patología en Estados Unidos. Por tanto, las posibles ventajas de la quimioterapia neo-adyuvante y las diferencias o equivalencias con respecto a la quimioterapia adyuvante en el tratamiento de los pacientes con CNMP deben de enmarcarse como un tema en debate para ser aclarado en estudios futuros.

\section{Conclusiones}

El discreto incremento en la supervivencia obtenido con la incorporación de la quimioterapia adyuvante en pacientes diagnosticados de CNMP localizado tras la resección quirúrgica ha sido confirmado de forma estadísticamente significativa. El beneficio por el momento parece más claramente relacionado con aquellos casos que presentan estadios patológicos II y III así como con la utilización de combinaciones de cisplatino y vinorelbina, independientemente de la edad, del sexo o del tipo de cirugía realizada. Sin embargo, debido a su potencial toxicidad dicho tratamiento debe ser ofrecida solamente a aquellos pacientes que cuentan con un buen estado funcional tras la cirugía. Queda por aclarar el papel que juega la administración de otros agentes citotóxicos o combinaciones, así como el de la radioterapia con las técnicas nuevas disponibles y si existen diferencias entre la administración previa o posterior a la cirugía. De forma paralela, un concepto más vanguardista representativo del futuro oncológico, va dirigido hacia la investigación de características biológicas y fármaco-genómicas que definan con precisión a los pacientes que verdaderamente se benefician de un tratamiento adicional así como el tipo de agente a utilizar bien por su efecto citotóxico, biológico o inmunológico específico, empleados de forma aislada o secuencial.

Correspondencia:

Dr. E. Esteban

Servicio de Oncología Médica

Hospital Universitario Central de Asturias

Julián Clavería, s/n,

E-33006 Oviedo (Asturias)

eestebang@seom.org 


\section{Bibliografía}

1. Mountain CF. Revisions in the international system for staging lung cancer. Chest. 1997; 111: 1710-1717.

2. Non-Small Cell Lung Cancer Collaborative Group. A meta-analysis using updated data on individual patients from 52 randomized clinical trials. BMJ 1995; 311; 899-909.

3. Postoperative radiotherapy in non-small cell lung cancer: systematic review and meta-analysis of individual patient data from nine randomised controlled trials. PORT metaanalysis Trialists Group. Lancet 1998; 352, 257-263.

4. Wada H, Hitomi S, Teramatsu T, et al. Adjuvant chemotherapy after complete resection in non-small cell lung cancer. J Clin Oncol 14: 1048-1054, 1996.

5. Tada H, Yasumitsu T, luchi K, et al. Randomized study of adjuvant chemotherapy for completely resected non-small cell lung cnacer. Proc J Clin Oncol 2002; 21: 313a (abstr 1250).

6. Kato H, Ichinose Y, Ohta M, et al. A randomized trial of adjuvant chemotherapy with uracil-tegafur for adenocarcinoma of the lung. N Engl J Med 2004; 350: 1713-1721.

7. Wada H, Miyahara R, Tanaka F, et al. Postpoerative adjuvant chemotherapy with PVM and UFT a randomized clinical trial: West Japan Study Group for lung cancer surgery. Eur J Cardiothorac Sug 1999; 14: 438-443.

8. Endo C, Saito Y, Iwanami H, et al. A randomized trial of postoperative UFT therapy in p stage I, II non-small cell lung cancer: North-east Japan study group for lung cancer surgery. Lung Cancer 2003; 40: 181-186.

9. Imaizumi M. A randomized trial of postoperative adjuvant chemotherapy for p-stage I non-small cell lung cancer (4th cooperative study). Lung Cancer 2003; 41: S2 (abstr 0180).

10. Hamada C, Tanaka F, Ohta M, et al. Meta-analysis of postoperative adjuvant chemotherapy with tegafur-uracil in non-small cell lung cancer. J Clin Oncol. 2005; 23: 49995006.

11. Keller Sm, Adak S, Wagner H, et al. A randomized trial of postoperative adjuvant therapy in patients with completely resected stage II or III non-small cell lung cancer. N Engl J Med. 2000; 343: 1217-1222).

12. Waller D, Peaje MD, Stephens RJ, et al. Chemotherapy for patients with non-small cell lung. The surgical setting of the Big Lung Trial. Eur J Cardio Thorac Surg. 2004; 26, 173-182).

13. Scagliotti GV, Fossati R, Torri V, et al. Randomized study of adjuvant chemotherapy for completely resected stage I, II, or IIIA non-small cell lung cancer. J Nat Cancer Inst 2003; 95: 1453-1460.

14. The Internacional Adjuvant Lung Cancer Trial Collaborative Group. Cisplatin-based adjuvant chemotherapy in patients with completely resected non-small-cell lung cancer. N Engl J Med. 2004; 350: 351-360.

15. Winton $\mathrm{T}$, Livingston $\mathrm{R}$, Jonson $\mathrm{D}$, et al. Vinorelbine plus cisplatin vs observation in resected non-small-cell lung cancer. N Engl J Med 2005; 352: 2589-2597.
16. Pepe C, Hasan B, Winton T, et al. Adjuvant chemotherapy in elderly patients: An análisis of National Cancer Institute of Canada Clinical Trials Group and Intergroup BR.10. Proc Am Soc clin Oncol 2006; 24: 366s (abstr. 7009).

17. Strauss GM, Herndon J, Maddaus MA, et al. Randomized clinical trial of adjuvant chemotherapy with paclitaxel and carboplatin following resection in stage IB non-small cell lung cancer: Report of Cancer and Leukemia Group B. Protocol 9633. Proc Am Soc Clin Oncol. 2004; (suppl 14): S621.

18. Strauss GM; Herndon JE, Maddaus MA, et al. Adjuvant chemotherapy in stage IB non-small cell lung cancer: Update of Cancer and Laukemia Group B (CALGB) protocol 9633. Proc Am Soc clin Oncol 2006; 24: 365s (abstr. 7007).

19. Douillar J, Rosell R, Delena M, et al. ANITA: Phase III adjuvant vinorelbine and cisplatin versus observation in completely resected (Stage I-III) non-small cell lung cancer patients: Final results after 70-months median followed. J Clin Oncol 2005; 23 (suppl 16S): 624.

20. Pignon JP, Tribodet H, Scagliotti GV, et al. Lung Adjuvant Cisplatin Evaluation (LACE): A pooled analysis of five randomized clinical trials including 4.584 patients. Proc Am Soc Clin Oncol 2006; 24: 366s (abstr. 7008).

21. Soría J, Haddad V, Olaussen KA, et al. Inmunohistochemical staining of the excision repair cross-complementing 1 (ERCC1) protein as predictor for benefit of adjuvant chemotherapy in the international Lung Cancer (IALT). Proc Am Soc Clin Oncol 2006; 24: 366s (abstr. 7010).

22. Kelly K, Gaspar L, Chansky K, et al. Low incidence of pneumonitis on SWOG 0023: A preliminary analysis of an ongoing phase III trial of concurrent chemoradiotherapy followed by consolidation docetaxel and Iressa/placebo maintenance in patients with inoperable stage III non-small cell lung cancer. J Clin Oncol. 2005; 23 (suppl 16S): 634.

23. Butts C, Murria N, Maksymiuk A, et al. Randomized phase IIB trial of BL25 liposome vaccine in stage IIIB and IV non-small-cell lung cancer. J Clin Oncol 2005; 23: 66756681.

24. Vansteenkiste J, Zielinski M, Dahabre J, et al. Multi-center, double-blind randomized, placebo-controlled phase II study to assess the efficacy of recombinant MAGE-A3 vaccine as adjuvant therapy in stage IB/II MAGE-A3-positive, completely resected, non-small cell lung cancer. Proc Am Soc Clin Oncol 2006; 24: 368s (abstr. 7019)

25. Depierre A, Milleron B, Moro-Sibilot D, et al. Preoperative chemotherapy followed by surgery compared with primary surgery in respectable stage I (except T1N0), II and IIIA NSCLC. J Clin Oncol 2002; 20: 247-253.

26. Pisters K, Vallieres E, Bunn P, et al. S9900: A phase III trial of surgery alone or surgery plus preoperative paclita$\mathrm{xel} /$ carboplatin chemotherapy in early stage non-small cell lung cancer: Preliminary results. J Clin Oncol 2005; 23(suppl 16): S624. 